\title{
A new type of biomimetic fish with oscillatory propulsion method
}

\author{
Zhang Lei, Deng Yongchao*, Li Kunlun, Qiu Linchuan \\ School of Physics and Electronics, Henan University
}

\begin{abstract}
The traditional multi joint mechanical serial parallel structure biomimetic fish has lower propulsive efficiency, more energy transmission loss, more complicated mechanical design and control algorithms because of more mechanical joints. While the flexible body has infinite degrees of freedom, and the energy in it is stored by elasticity and inertia, it is more viscous than that of multi joints. This project studies the principle of carangidae fish in carangidae carp, takes it as the bionic object, and designs a biomimetic fish with a flexible body tail structure to improve propulsive efficiency. This study explores the biomimetic fish propulsion speed and caudal aspect ratio, caudal oscillation frequency, modal distribution curve, and optimizes a parameter set of fish tail that can achieve effective promotion. So it is good for the exploration of the development of high speed, high efficiency, high maneuverability of bionic propeller swing on theoretical basis and practical aspects.
\end{abstract}

Keywords: bionic propulsion; oscillating propulsion; fluid-structure interaction system; propulsive performance

\section{Introduction}

Underwater vehicle plays an important role in marine resources exploration, underwater target search and rescue and military investigation. However, the traditional thruster based on propeller mechanical structure has the defects of high noise, poor maneuverability and low propulsive efficiency[1]. In addition, the propeller is easily entangled by debris, resulting in the loss of propulsion power. Therefore, it is significant to study the propulsion of new propulsion methods. After millions of years of natural selection and evolution, fishes form unique patterns and movements to suit for the complex underwater environment. These movements are mainly divided into the body / caudal fin propulsion mode and the central fin / fin propulsion model[2]. The propeller based on BCF type of fish with exercise using caudal and pectoral not only has high mobility but also has very good propulsive efficiency. For instance, the speed of BCF type of tuna in still water can be $80 \mathrm{~km} / \mathrm{h}$, promoting the efficiency almost to $90 \%$, while the carangidae mode of carp startup fast, acceleration - close to $50 \mathrm{~m} / \mathrm{s}[3]$. Due to the excellent underwater movement ability of fish, many propulsion models of biomimetic propulsion have been studied by many researchers, and a series of important achievements have been achieved[4]. With the development of bionics[5], fluid mechanics and material science, it can be predicted that the biomimetic fish like robot has the advantages of high efficiency, high concealment and high maneuverability compared with the propeller.

\section{Biomimetic fish prototype design and manufacture}

\subsection{An external 3D model of biomimetic fish and caudal fin design}

This design based on the theoretical and experimental study of CARANGOID fish body / tail fin propulsion mode[6], is to improve the biomimetic fish propulsion efficiency. The main source of fish propulsion comes from its body that $1 / 3$ after caudal part. So in the design of the fish, the front $2 / 3$ part of the fish body is designed as a rigid structure[7], while the $1 / 3$ part uses silica gel mold into a flexible structure with a certain rigidity, and the tail is set to be mixed with flexible structure.

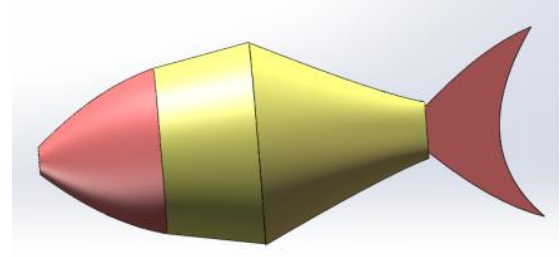

Fig.1. external structure of biomimetic fish

After hundreds of millions years of evolution, fishes have different tail fins of different shapes and flexibility[8], and the shape of the caudal fin is often represented by a formula (1):

$$
P=L / S
$$

Where $\mathrm{P}$ is the aspect ratio and $\mathrm{L}$ is the tail fin extension, and $\mathrm{S}$ is the caudal fin area. The results show that the speed of bionic tuna propulsion is high[9], but not necessarily the best propulsion efficiency. This shows that the influence of the ratio of the tail to the chord on the speed and propulsive efficiency of the fish is not a simple linear relationship. In this case, five different aspect ratio fins are designed as a reference. Through the actual measurement of carp body proportion parameters, 
when the fish body length was $230 \mathrm{~mm}$, the average length of the caudal fin show is about $70 \mathrm{~mm}$. The caudal fin models with the aspect ratios 2.3, 3.4, 3.6, 4.2, and 6.3. Comparing with the corresponding real caudal fin, as Fig. 2.

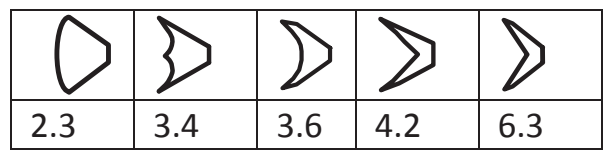

Fig. 2. Shape of caudal fin with different aspect ratio

Flexible tail fin structure. In this study, the flexible body fishtail is made of silica gel, and the tail handle is made of rigid PLV material. The two components constitute a flexible and rigid tail structure: (1) ensure that the hardness of the tail of the flexible body can handle the pressure underwater without deformation in movement; (2) ensure the flexible body has a certain degree of hardness, and not hinder the movement of the structure of swing.

\subsection{Biomimetic fish internal power unit and transmission structure}

According to the principle of electromagnetic induction, the conductor will produce inductive magnetic field when it is energized[10]. The magnet produces a strong magnetic field, the hollow coil is fixed in the structure with freedom in the horizontal direction only. When the current passes through the hollow coil, the coil is deflected by the torque in the horizontal direction. So we design the swing structure of the magnet and the hollow coil according to above principles, with two magnetic poles being opposite, hollow coil in the magnet middle. When the magnitude and direction of the current in the coil are changed, the coil can swing the swinging rod horizontally in the horizontal direction.
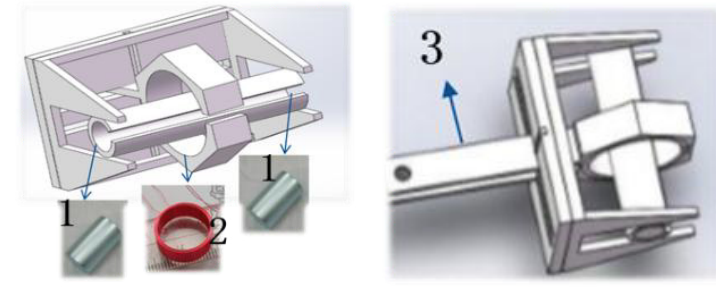

1:magnet; 2: hollow coil; 3 : swinging rod

Fig. 3. Schematic diagram of oscillating device

For a single joint body / tail fin like biomimetic fish, the tail fin swing translation complex motion is the first consideration in the design of biomimetic fish. Therefore, according to the relevant experimental conclusions, the following parameters are set for flexible tail fin:

(1) Caudal to the maximum angle of attack: $\alpha_{\max } \in$

$\left(15^{\circ}, 25^{\circ}\right)$

(2) The aspect ratio of caudal fin is: $\mathrm{D}=2.3, \mathrm{D}=4.2$, $\mathrm{D}=6.3$.

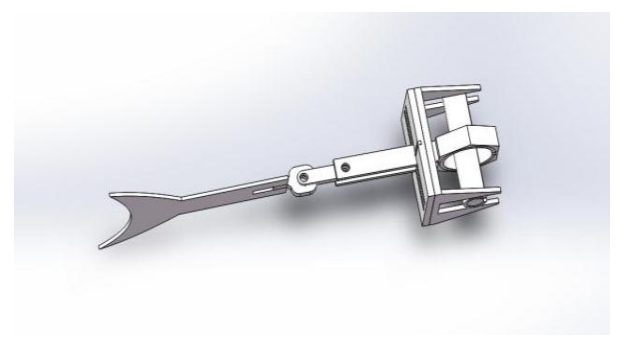

Fig. 4. Schematic diagram of driving structure of tail fin double pendulum lever

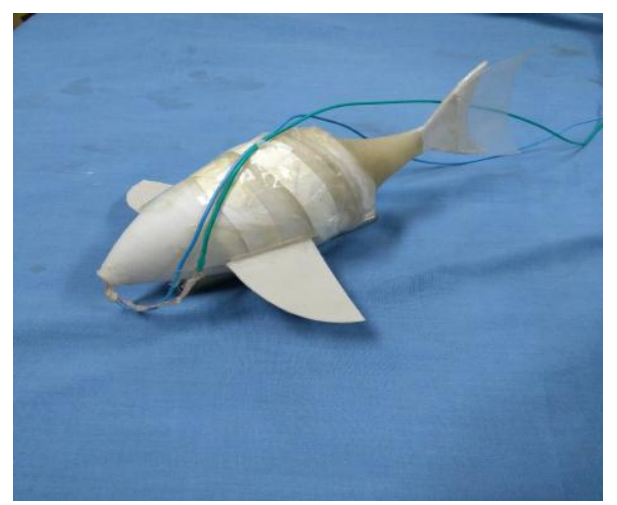

Fig. 5. The final biomimetic fish model

In Fig. 4, three micro bearings are respectively placed in three circular through holes. The tail fin and the frame part are fixed by silica gel to form two fixing points, and the connecting rod between the two bearings is a connecting rod, and the whole structure is composed of a double pendulum rod structure[11]. When the coil is energized, the coil will deflect under the action of the magnetic field force to drive the oscillating coil pendulum rod, and then drive the tail fin swinging rod through the connecting rod to realize the transmission. In this process, the coil and swing rod belong to the active rod, and the tail fin and pendulum rod belong to the driven rod; on the contrary, when the coil power belongs to the active flexible tail fin, pendulum rod coil pendulum belongs to the follower rod. The double pendulum transmission structure makes full use of the flexible body to store and dissipate energy and improve energy efficiency. Figure 5 shows the final biomimetic fish model

\section{Control system design}

The driving device of the tail fin is to control the magnitude and direction of the current in the coil by PWM signal. Therefore, the essence of the control of the biomimetic fish is the control of the duty ratio and the frequency of the PWM signal. Biomimetic fish control system is a relatively complex system, which needs to control the output of the signal, data transmission and data analysis simultaneously. So it requires close integration of multiple functional modules and execution in accordance with certain processes. The control system adopts the top-down design idea of multiple modules. 
The flow chart is shown in Figure 6.

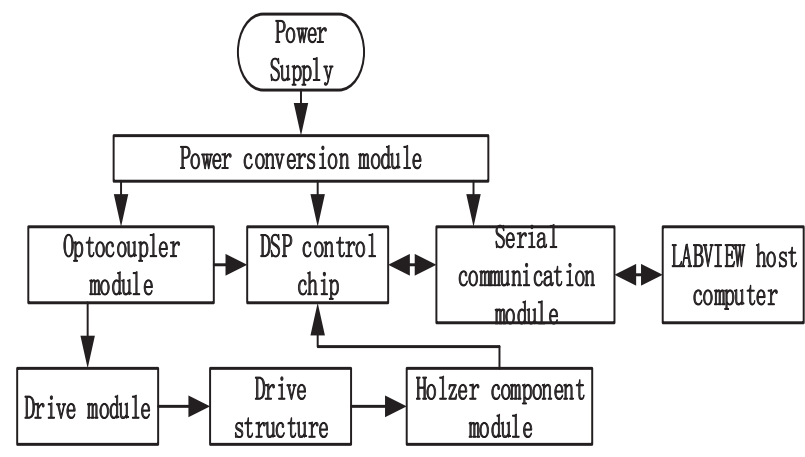

Fig. 6. Flow chart of biomimetic fish control system

\section{Experiment}

According to the characteristics of the biomimetic fish swimming, in order to ensure the independence movement of the biomimetic fish and reduce experimental device caused on the result of the measurement error, the experimental design uses the image resolution of $640 * 480$ camera to shoot biomimetic fish swimming under water. The image processing method is used to intercept the image in the computer. We identify and track the biomimetic fish through target recognition and template matching, and record the position coordination of the biomimetic fish, and then draw the trajectory curve. Finally, we calculate the swimming speed of the biomimetic fish.

\section{Results and discussion}

5.1. The relationship between the oscillating frequency and the velocity of the caudal fin

According to the characteristics of carangidae fish tail fin, we select features for maximum swing of 25 degrees and aspect ratio were 4.2 and 2.3 , the flexible fin structure 6.3. In this experiment, two cycles of PWM wave are used, one of which is duty cycle $\mathrm{S}=50 \%, \mathrm{~T}=64 \mathrm{~s}$ and the other duty cycle is $\mathrm{S}=90 \%, \mathrm{~T}=64 \mathrm{~s}$ The tail fin oscillation frequency is $1-9 \mathrm{hz}$.

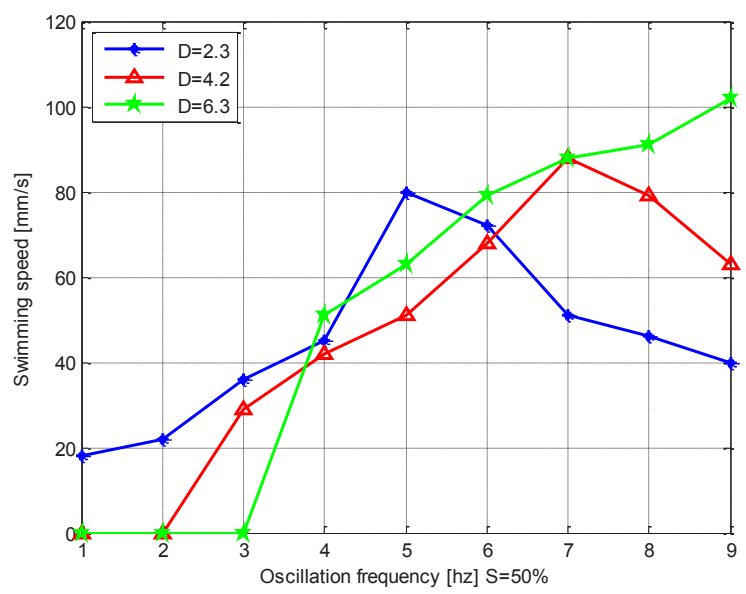

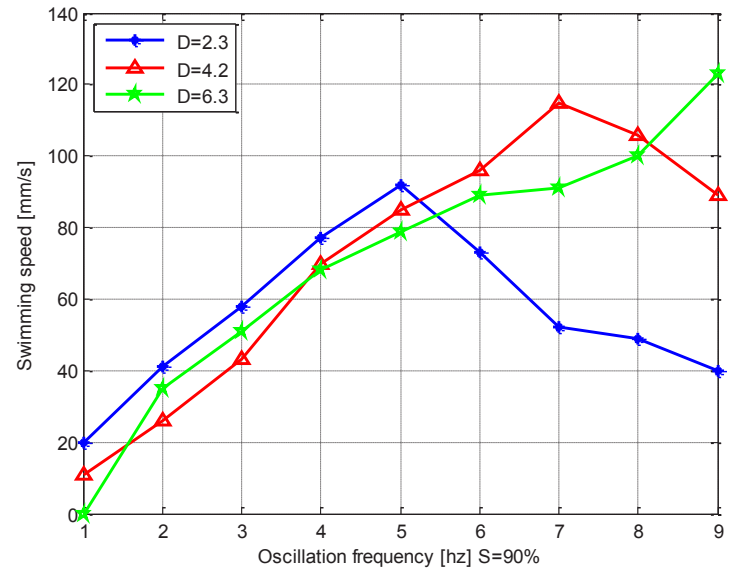

Fig. 7. Relation curve between tail fin oscillation frequency and fish speed

As shown in Figure 7 above:

(1). For a tail with arbitrary aspect ratio, its moving speed follows a two curve relationship with the oscillation frequency, and reaches its maximum near a certain frequency.

(2). When the aspect ratio is relatively small, the motion velocity following the frequency change gently and distributes evenly.

(3). When the aspect ratio is relatively large, the motion speed following the frequency change rapidly, but the overall change range is small.

\subsection{The curvilinear relation between input power and speed}

In this measurement experiment, the input voltage of the system is $E=6.5 \mathrm{v}$, the tail fin oscillation frequency is $6 \mathrm{hz}$, the PWM duty cycle of the control signal is from $10 \%$ to $90 \%$, and the duty cycle is increased by $10 \%$ each time, and the experiment is carried out once. The relationship between the input power and the swimming speed is shown in Figure 8.

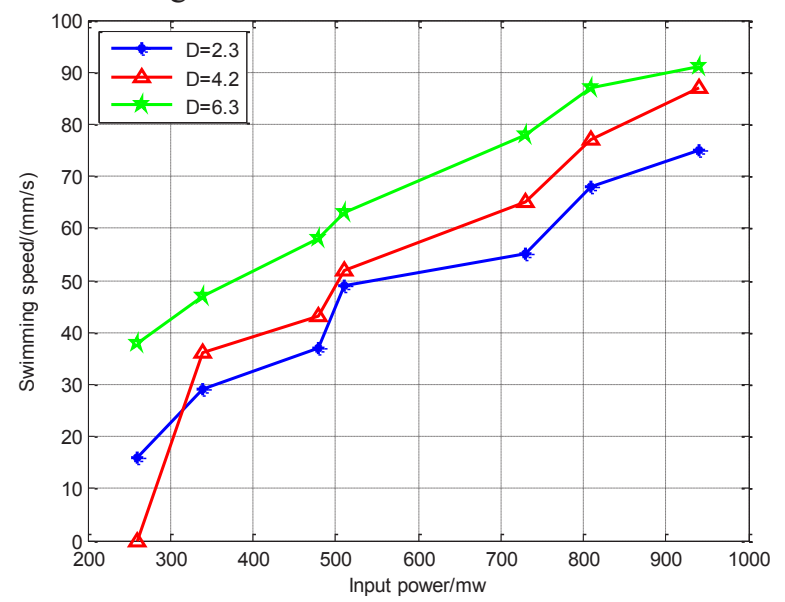

Fig. 8. Relationship between input power and fish speed

As shown in Fig. 8, in a certain range, the input power of the biomimetic fish with any aspect ratio of caudal fin has a positive correlation with the swimming speed of fish. When the input power increases, the swimming 
speed of fish will increase, and the smaller the aspect ratio, the greater the influence of input power on the speed of biomimetic fish swimming.

5.3. The relationship between the oscillating frequency of the caudal fin and the propulsive efficiency

The propulsive efficiency of biomimetic fish is determined by various parameters. But in the same experimental environment[8], the coefficient of fluid resistance, the density of fluid and the surface area of fish are small, so they can be regarded as constants[9]. Now the product of $\mathrm{c}$ is the product of the three. The propulsive efficiency of the robotic fish can be expressed by expressions (1), (2) and (3):

$$
\begin{gathered}
P=\frac{1}{2} \rho C_{d} S_{V}^{2} \bullet V \\
c=\frac{1}{2} \rho C_{d} S \\
\eta=\frac{P}{U I}=\frac{c V^{3}}{U I} \times 100 \%
\end{gathered}
$$

Where $P$ is the effective power, $\rho$ is the density of water, and $C_{d}$ is the drag coefficient. Where $S$ is the cross section, $U$ is the voltage at both ends of the oscillating device, and $I$ is the current in the hollow coil. Where $\eta$ is the efficiency of converting electric energy into kinetic energy on the biomimetic fish, $V$ is the biomimetic fish swimming speed. In this experiment, the driving signal PWM duty cycle is $90 \%$, the corresponding system input voltage is $5.6 \mathrm{v}$, the system input current is $168 \mathrm{~mA}$, and the system input power is $940.8 \mathrm{~mW}$. The aspect ratio of flexible biomimetic fish fin is $D=2.3,4.2$ and 6.3 respectively.

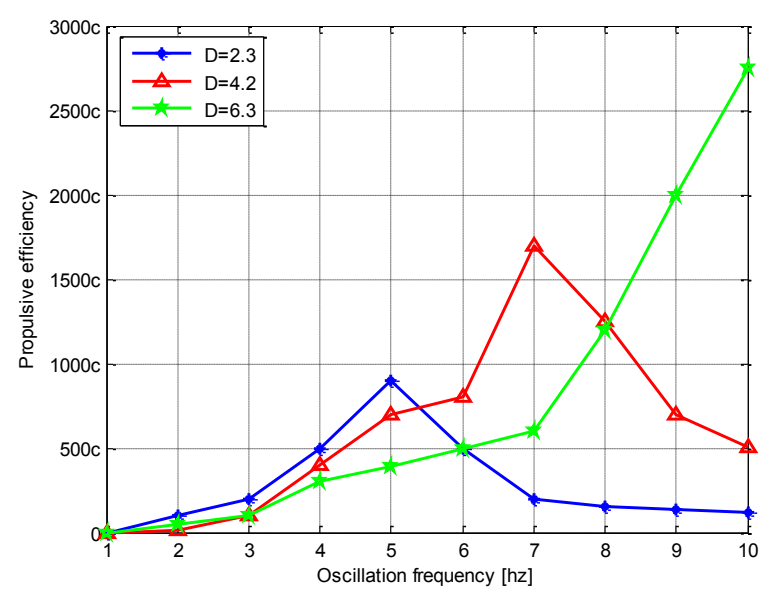

Fig. 9. Relationship curve of tail oscillation frequency and propulsive efficiency

As can be seen from Fig.9, (1) biomimetic fish propulsion efficiency with oscillating frequency increases and then decreases, the propulsion efficiency reaches the maximum value at a certain frequency. (2) under the same condition, when the ratio of the fin to tail ratio affects the propulsive efficiency of the biomimetic fish, the oscillation frequency of the caudal fin and the ratio of the aspect ratio of the tail fin are greater, and the oscillation frequency of the fish when the fish reaches the maximum propulsive efficiency is greater. (3) within a certain range, the greater the tail fin aspect ratio, the greater the propulsive efficiency of the biomimetic fish.

\section{Conclusions}

In this study, a biomimetic fish with flexible fish tail was designed to reproduce the complex motion of fish, and the influence of parameters of different flexible caudal fin on propulsion speed and propulsive efficiency was discussed. For a tail with arbitrary aspect ratio, its moving speed follows a two curve relationship with the oscillation frequency, and reaches its maximum near a certain frequency. In a certain range, the input power of the biomimetic fish with any aspect ratio of caudal fin has a positive correlation with the swimming speed of fish. For flexible fish body system, the effect of increasing the swing frequency or simply increasing the input power on the propulsion speed is not complete. In order to get the best propulsion efficiency, we should balance the tail fin ratio, swing frequency, input power and other factors, to maximize the speed of fish movement, in order to achieve high performance propulsion.

\section{Acknowledgements}

This work has been supported by the Foundation of Key science and technology research projects of Education Department of Henan Province (CX0001F01158).

\section{References}

1. J. Edward Colgate, Kevin M. Lynch, Mechanics and Control of Swimming: A Review, IEEE Journal of Oceanic Engineering, 2004, 29(3): pp. 660-673.

2. M. Sfakiotakis, D. M. Lane, and J. B. C. Davies. Review of fish swimming, modes foraquatic locomotion. IEEE Journal of Oceanic Engineering, 1999, 24(2): pp. 237-252.

3. J. Deng, X. M. Shao, Z. S. Yu. Hydrodynamic Studies on Two Traveling Wavy Foils in Tandem Arrangement. Physics of Fluids. 2007, 19 : pp. 1-10.

4. J.D.Liu, H.S.Hu.Mimicry of Sharp Turning Behaviours in a Robotics Fish. Proceedings of the 2005 IEEE, International Conference on Robotics and Automation, Barcelona, Spain, April ,2005.

5. J.E dward Colgate, Kevin M.Lynch.Mechanics and Control of Swimming:A Review. IEEE Journal of Oceanic Engineering.2004,29(3):660 669.

6. G. Buccino, et al.Action Observation Activities Premotor and Parietal Areas in a Somatotopic MannerEuropean Journal of Neuroscience 2001(13):400 404 .

7. Shinjo N, Swain GW.Use of a Shape Memory Alloy for the Design of an Oscillatory Propulsion System[J]. 2004 IEEE Journal of Oceanic 
Engineering, 2004, 29(3): 750 755.

8. B. E. Flammang, G. V. Lauder, D. R. Troolin and T. Strand. Volumetric imaging of shark tail hydrodynamics reveals a three-dimensional dual-ring vortex wake structure. Proceedings of the Royal Society, Biological Sciences, 2011,5 :pp. 1-9.

9. Valdivia y Alvarado, P. and K. Youcef-Toumi, Design of Machines with Compliant Bodies for Biomimetic Locomotion in Liquid Environments, ASME Journal of Dynamic Systems, Measurement, and Control 128, March 2006: pp. 3-13.

10. Tayebi A, Mcgilvray S, Roberts A, et al. Attitude estimation and stabilization of a rigid body using low-cost sensors[C]. Decision and Control, 2007, IEEE Conference on. IEEE, 2008:6424-6429.

11. Bouabdallah S, Becker M, Siegwart R. Autonomous miniature flying robots: coming soon - Research, Development, and Results[J]. Robotics \& Automation Magazine IEEE, 2007, 14(3):88-98. 